\title{
Correlative Analysis Between Severity of Urolithiasis and Laboratory Parameters and Its Implication in Evaluation of the Probable Risk Profile
}

\author{
Rajeev T.Pa, Yashasvi Singha, b, Sasanka Kumar Barua ${ }^{a}$, \\ Debanga Sarma ${ }^{a}$
}

\begin{abstract}
Background: Urolithiasis presents serious hazard which significantly elevates the cost of national health expenditure in almost every part of both the hemispheres. There is high risk of hospitalization with loss of valuable human resource and decreased productivity along with it. Risk factors still evade the exact etiology and search for optimal serum panel is still in its infancy. Urolithiasis incidence has gradually increased in last 3 decades which suggests that some constant metabolic and urinary parameters are implicated in the risk of occurrence of urinary stone. The present study is intended to identify a panel of serum parameters, urinary parameters, radiological characteristics and correlating it with the clinical severity of stone disease.
\end{abstract}

Methods: The present study was conducted at the Department of Urology at GMCH Guwahati. The authors retrospectively analyzed 151 patients undergoing stone surgery from a period of January 2016 to August 2017. Data comprised of all serum and urinary examinations done 1 week preoperatively and radiological scans within 1 month before surgery. Spearman test was used to determine correlation and analysis of variance (ANOVA) was applied for comparison between more than two categories.

Results: Stone multiplicity was positively correlated with upper tract stone sides $(\mathrm{r}=0.530, \mathrm{P}<0.01)$, large stone volume $(\mathrm{r}=0.172, \mathrm{P}<$ $0.02)$, stone recurrence, urinary infection and urine protein. Upper tract stone sides number was positively correlated with upper tract obstruction sides $(\mathrm{r}=0.542, \mathrm{P}<0.03)$, large stone volume $(\mathrm{r}=-0.321$, $\mathrm{P}<0.01)$. Upper tract obstruction sides number was positively correlated with large stone volume $(\mathrm{r}=-0.848, \mathrm{P}<0.01)$.

Conclusions: Results demonstrated that urinary tract obstruction and total stone volume significantly correlated with abnormal serum panel, urinary profile and were harbinger of complex stone pattern.

Manuscript submitted February 5, 2018, accepted February 26, 2018

aDepartment of Urology, 3rd Floor, GMCH Complex, GMC Hostel Road , Bhangagarh, Guwahati, 781032, Assam, India

${ }^{b}$ Corresponding Author: Yashasvi Singh, Department of Urology, 3rd Floor, GMCH Complex, GMC Hostel Road, Bhangagarh, Guwahati, 781032, Assam, India. Email: Yashasvisingh075@gmail.com

doi: https://doi.org/10.14740/wjnu332w
Keywords: Hypertension; CKD; Stone multiplicity; Upper tract obstruction

\section{Introduction}

Urolithiasis is an omnipresent pandemic disorder affecting large patient population worldwide and especially in South Asia. About $10 \%$ of people will experience nephrolithiasis in their lifetime and about $50-70 \%$ of those will have recurrences $[1,2]$. Urolithiasis prevalence has been on an upswing in both sexes and in certain areas of the Indian subcontinent like NE India; the lifetime hazard appears to be even higher. A lot of capital has been used in the overall management of urinary stones worldwide but its forestalling has been a spurned field. A recent study based upon the National Health and Nutrition Examination Survey (NHANES) estimated that $19 \%$ of men and $9 \%$ of women will be diagnosed with a kidney stone by the age of 70 years [3]. Recurrence rates of renal stone are approximately $10 \%$ per year, $50 \%$ over a period of $5-10$ years and $75 \%$ over 20 years period. The incidence rate of nephrolithiasis varies with geographical region of an individual country. Nearly 2 million people in India are affected with urolithiasis every year and many parts of the country have names denoted as a stone belt that is, Gujarat, Maharashtra, Punjab, Rajasthan, Delhi, Haryana and part of states on Northeast side[4]. There are a number of epidemiological arenas consisting of anatomic, metabolic, dietary and urinary factors that incline to the development of urolithiasis. The frequency with which these different risk factors occur in patients with recurrent stone disease and the role of genetic susceptibility are reviewed at the end of this topic. Upper urinary tract stones may lead to hydronephrosis and renal compromise. Nonetheless, hypertension, diabetes mellitus and chronic kidney disease (CKD) were proved to be correlated with nephrolithiasis. Many authors agree and it is our firm belief that stones in patients with CKD should be cleared [5]. However, the risk factor that may influence the calculus kidney damage is still unclear and needs further evaluation.

Nearly $75 \%$ of patients with nephrolithiasis form calcium stones mostly comprising of calcium oxalate or calcium phosphate to some extent, while uric acid stones make up less than 10 percent of all stones [6]. Renal stones consist of a variety of 
crystalline and non-crystalline materials, knowledge of which influences clinical decision. Analysis of $24 \mathrm{~h}$ urine composition provides data on the possible contents of a stone but does not perfectly predict stone type. The frequent crystalline materials found in kidney stones are calcium oxalate, calcium phosphate, uric acid, and struvite though mutual coexistence for them in most cases has become an undeniable fact. Non-crystalline materials found in stones include blood and protein. The aim of this study is to establish a correlation between different calculus clinical patterns and laboratory parameters in patients with urolithiasis, in order to further explore the mechanism of these parameters changes after stone formation and also to find out some risk factors of kidney damage after urolithiasis occurs.

\section{Methods}

This retrospective study included 151 patients with urolithiasis who underwent surgery for renal, ureteric and vesical stones at the Department of Urology and Renal Transplantation between January 2016 to August 2017 and were in follow-up for at least 6 months in the Urology OPD. Diagnosis of stone diseases and their clinical implication were assessed by USG W/A, intravenous urogram (IVU), Non-contrast CT kidney-ureter-bladder scanning (NCCT KUB) and contrast-enhanced computed tomography (CECT (W/A + pelvis + urography) ) when required accordingly. "Stone multiplicity" was construed as multiple urinary tract stones present, disregarding their locations. "Upper urinary tract obstruction" was characteristically present when ureteric calculi or hydronephrosis caused by renal calculi were diagnosed. "Large stone bulk" was defined as stone's size greater than $2 \mathrm{~cm}$ in diameter. Data on patient's age at presentation, gender, co-morbidities, past medical and surgical history were recorded. Diagnosis of uncontrolled hypertension and diabetes mellitus was based on medical history and current use of medication. Height and weight were measured and body mass index was calculated.

All hematological and biochemical test results were obtained preoperatively, within 1 week before primary surgery. Authors evaluated the serum levels of urea, creatinine, sodium, potassium, magnesium, calcium, phosphorus, alkaline phosphate, uric acid and albumin. Patients were asked to collect morning urine samples which were examined for $\mathrm{pH}$, specific gravity, protein and bacteria. Patients with borderline renal function and objective evidence of CKD on USG W/A upfront underwent $99 \mathrm{~m}$ Tc-DTPA renal scan. Split GFR below 20\% than that of the contralateral kidney was considered as "mild damage" and above $20 \%$ as "moderate damage". Ipsilateral kidney's GFR below $10 \mathrm{~mL} / \mathrm{min}$ was considered as "severe damage". Discrete and continuous variables were compared using Chi-square test, Student's $t$-test and post-hoc analysis respectively and correlation coefficient was calculated using the Pearson's R analysis. $P$ values less than 0.05 were considered statistically significant. SPSS version 21.0 was used to evaluate all statistical analysis.

\section{Results}

The median age for the entire cohort was $55.15 \pm 14.26$ years
Table 1. Distribution Pattern of Categorical and Clinical Parameters With Overall Distribution

\begin{tabular}{lll}
\hline Age (years) & Mean \pm SD & $\mathbf{5 5 . 1 5} \pm \mathbf{1 4 . 2 6}$ \\
\hline Gender & Male/female & $87 / 64$ \\
Recurrence & Yes/no & $50 / 101$ \\
BMI & Mean \pm SD & $25.21 \pm 3.26$ \\
HTN & Present/absent & $48 / 103$ \\
DM & Present/absent & $29 / 122$ \\
CKD & Present/absent & $44 / 107$ \\
DM + CKD & Present/absent & $12 / 139$ \\
Stone multiplicity & Present/absent & $85 / 66$ \\
Upper tract stone site & $0 / 1 / 2$ & $32 / 62 / 57$ \\
Upper tract obstruction side & $0 / 1 / 2$ & $16 / 65 / 67$ \\
Large stone bulk & Present/absent & $41 / 110$ \\
Ipsilateral kidney damage & Mild/moderate/severe & $48 / 80 / 23$ \\
\hline
\end{tabular}

taking into account all the four respective groups but irrespective of gender group $(\mathrm{P}=0.034)$. Median age was maximal for the ureteric stone group (Table 1). While examining the biochemical parameters it was seen that median value of serum urea was maximum in ureteric stone group but the overall distribution did not reach significant level $(\mathrm{P}=0.950)$. Serum creatinine was analyzed in all the four groups as a constant variable both pre and postoperatively, and was maximal in the vesical calculus group when compared with other groups (P $=0.029)$ (Table 2). Protein metabolism that was specifically mapped upon in view of the dietary habits and serum albumin level was found to be significantly associated in all the four groups $(\mathrm{P}=0.012)$. Though serum calcium concentration holds an important position in urolithiasis development, our analysis could not get a significant association except in obstruction ( $\mathrm{P}$ $=0.001)$ group and large stone bulk group $(\mathrm{P}=0.002)$ for the same. Serum sodium $(P=0.032)$ and magnesium $(P=0.045)$ both were significantly associated with stone formation among all the four groups. Among these parameters, male and the aged were significantly associated with bladder calculi. Renal calculi were associated with recurrence, UTI and higher urinary protein, whereas ureteral calculi were associated with higher serum sodium level. The combination of renal and ureteric calculi was associated with higher serum creatinine. One third of the patient in the entire cohort had recurrence in the follow-up period (6 months) with maximum number of cases in the kidney stone group with significant association (Chi square value $=4.706, \mathrm{P}=0.019)$ and correlation $(\mathrm{r}=3.08, \mathrm{P}$ $=0.042)$. Hypertension was as significantly associated $(\mathrm{Chi}$ square value $=8.528, \mathrm{P}=0.001)$ and correlated $(\mathrm{r}=5.67, \mathrm{P}=$ $0.036)$ as recurrence in the four stone categories and was present in $31.79 \%$ cases. CKD was present in $29.14 \%$ cases when presented in isolation $(\mathrm{P}=0.043$, ) and in $7.94 \%$ cases when combined with DM II $(\mathrm{P}=0.404)$ which was not significant when present in combination (Table 3 ). Its correlation with the stone distribution groups did reach significant level $(\mathrm{P}=0.027)$ when analyzed statistically. Multiple stones were observed in $56.29 \%$ cases and were significantly associated (Chi square 
Table 2. Median Values of Continuous Variables With Corresponding P Values (Chi-Square Analysis/Pearson's R Analysis)

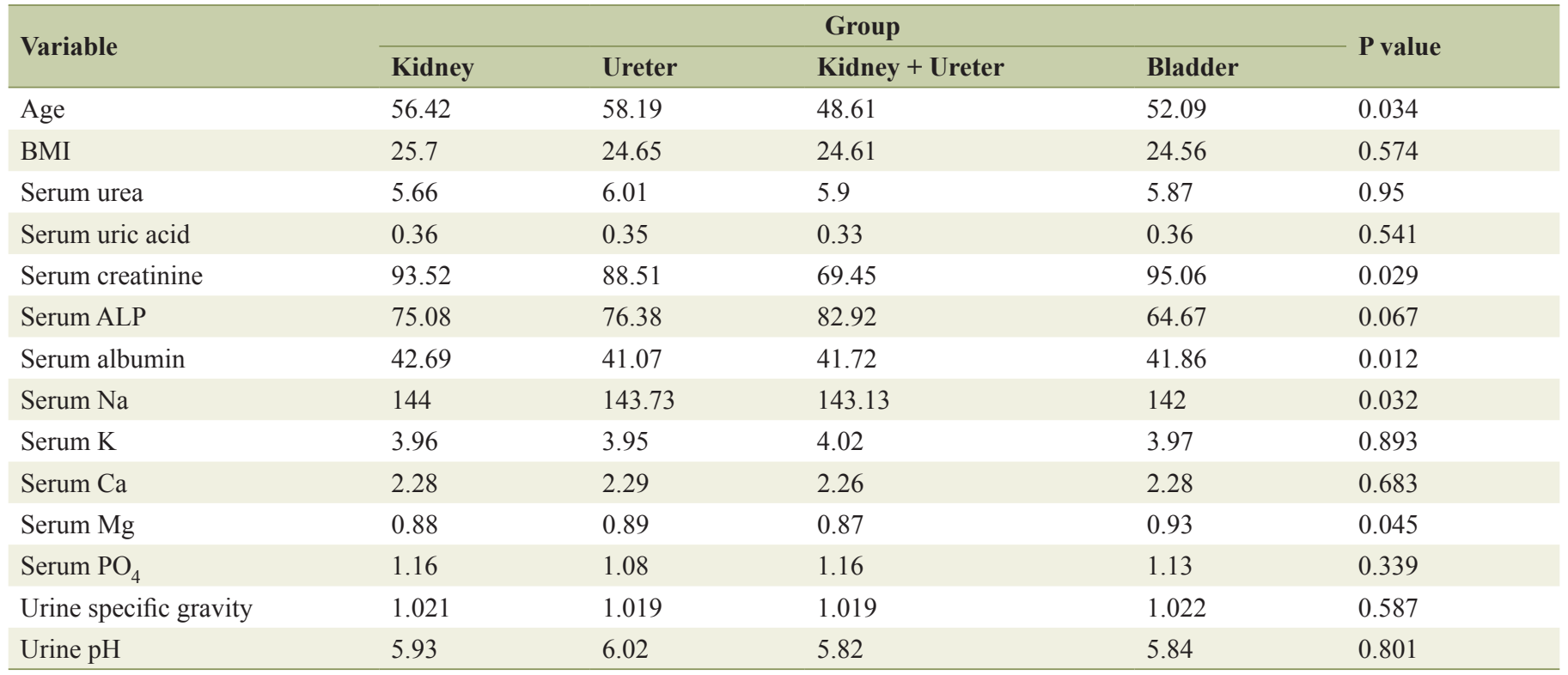

value $=8.348, \mathrm{P}=0.039)$ and correlated $(\mathrm{r}=-4.09, \mathrm{P}=0.027)$ with the stone groups. Multiple stones were managed in the same sitting if present on the same side and there was a gap of 3 to 6 months when present on the opposite side.

Upper tract stone sites incorporated $41.06 \%$ cases on a single site and $37.75 \%$ cases on two sites which was significantly associated (Chi square value $=14.743, \mathrm{P}=0.033$ ) and correlated $(r=-4.09, P=0.027)$ with the same. Hydronephrosis of the upper tract resulting from obstruction occurred in $43.04 \%$ on one side and $44.37 \%$ on both the sides and it was significantly associated (Chi square value $=14.81, \mathrm{P}=0.049$ ) and correlated $(\mathrm{r}=2.69, \mathrm{P}=0.022)$ with the four stone groups. Large stone bulk was seen in $27.15 \%$ and was not significantly associated and correlated with the same compelling on the importance of delay in presentation to the OPD. The time lag in presentation augmented the ipsilateral kidney damage which towered at $52.98 \%$ for moderate damage and $15.23 \%$ for severe damage and was significantly associated (Chi square value $=14.27, \mathrm{P}$ $=0.048)$ and correlated $(\mathrm{r}=3.10, \mathrm{P}=0.038)$ with the groups in consideration.

The correlation determination for stone multiplicity with other clinical variables revealed that stone multiplicity was positively correlated with upper tract stone sides $(\mathrm{r}=0.730, \mathrm{P}$ $<0.01)$, large stone volume $(\mathrm{r}=0.272, \mathrm{P}<0.02)$, stone recurrence $(\mathrm{r}=0.632, \mathrm{P}=0.001), \mathrm{CKD}(\mathrm{r}=0.062, \mathrm{P}=0.001)$, urine albumin $(\mathrm{r}=2.63, \mathrm{P}=0.012)$ and urine infection $(\mathrm{r}=1.80, \mathrm{P}$ $=0.001$ ). Upper tract stone sides number was positively correlated with age $(\mathrm{r}=2.32, \mathrm{P}=0.002)$, upper tract obstruction sides $(\mathrm{r}=0.742, \mathrm{P}<0.01)$, serum creatinine $(\mathrm{r}=4.67, \mathrm{P}=$ 0.002 ), serum sodium, and urinary infection, large stone volume $(\mathrm{r}=-0.321, \mathrm{P}<0.01)$, urine specific gravity $(\mathrm{r}=1.87, \mathrm{P}$ $=0.04)$ and urine infection $(\mathrm{r}=8.06, \mathrm{P}=0.003)$. Upper tract stone obstruction sides number was positively correlated with age $(\mathrm{r}=5.56, \mathrm{P}=0.033)$, BMI $(\mathrm{r}=9.87, \mathrm{P}=0.044)$, serum potassium $(\mathrm{r}=6.44, \mathrm{P}=0.021)$, serum creatinine $(\mathrm{r}=1.56, \mathrm{P}=$
$0.039)$, serum calcium $(\mathrm{r}=5.06, \mathrm{P}=0.001)$, serum phosphate $(\mathrm{r}=6.86, \mathrm{P}=0.042)$, urine albumin $(\mathrm{r}=0.443, \mathrm{P}=0.002)$ and urinary infection $(\mathrm{r}=0.443, \mathrm{P}=0.01)$, whereas negatively correlated with large stone volume $(\mathrm{r}=-0.748, \mathrm{P}<0.01)$ and serum magnesium $(\mathrm{r}=-4.67, \mathrm{P}=0.038)$. Large stone volume was positively correlated with age $(\mathrm{r}=0.879, \mathrm{P}=0.033)$, urine albumin $(\mathrm{r}=3.65, \mathrm{P}=0.011)$, serum phosphate $(\mathrm{r}=0.401, \mathrm{P}$ $=0.001)$, serum calcium $(\mathrm{r}=0.801, \mathrm{P}=0.002)$ and $\mathrm{CKD}(\mathrm{r}=$ $0.441, \mathrm{P}=0.023)$. Ipsilateral kidney damage was positively correlated with age, recurrence, CKD, hypertension, serum urea and serum creatinine, serum albumin (Table 4).

\section{Discussion}

In our patient population, bilateral upper tract calculi are correlated with higher serum sodium. Increased daily sodium intake tends to increase serum and urinary sodium in a significant manner which not only increases the net calcium excretion in the urine, but also increases urinary $\mathrm{pH}$ and decreases citrate excretion at the same time. Keeping the aforementioned aspect in consideration, the urinary concentration of calcium phosphate and monosodium urate also increased significantly. Thus, a high sodium intake was directly responsible for the crystallization of calcium salts in urine. Moreover, in our study, upper urinary tract obstruction side number was correlated with higher serum potassium, calcium and phosphate level, as well as lower magnesium level.

Calcium urolithiasis has hypercalcemia and hypercalciuria both as compelling and estimable factors requiring urgent and immediate action. Hypercalcemia exists when serum calcium level increases, together with measuring and calculation of physiologically active calcium when there is difference in the $\mathrm{pH}$ of blood or serum albumin [7]. In serum, calcium is either bound to albumin or is found in its free form (ionized). 
Table 3. Significant Association and Correlation Parameters of Categorical Variables and Clinical Parameter Groups

\begin{tabular}{lllll}
\hline Variable & Chi square coefficient & P value & Pearson r coefficient & P value \\
\hline Gender & 7.28 & 0.063 & -0.015 & 0.859 \\
Recurrence & 4.706 & 0.019 & 3.08 & 0.042 \\
HTN & 8.528 & 0.001 & 5.67 & 0.036 \\
DM II & 5.635 & 0.302 & 0.01 & 0.907 \\
CKD & 8.66 & 0.043 & 7.42 & 0.027 \\
DM + CKD & 2.92 & 0.404 & -0.002 & 0.979 \\
Multiple stones & 8.348 & 0.039 & -4.09 & 0.027 \\
Upper tract stone side & 14.743 & 0.033 & -0.858 & 0.041 \\
Upper tract obstruction side & 14.81 & 0.049 & 0.269 & 0.022 \\
Large stone bulk & 2.072 & 0.558 & 0.021 & 0.798 \\
Ipsilateral kidney damage & 14.27 & 0.048 & 3.1 & 0.038 \\
\hline
\end{tabular}

It is the ionized calcium which is of concern because ionized calcium is the physiologically active form of calcium as calcium bound to albumin is inactive. Standard lab tests usually measure the total calcium. When albumin is low, patients have lower total calcium on lab tests. Hence, patients with lower serum albumin tended to have higher serum active calcium level.
When the serum is alkaline, hydrogen ions bound to negatively charged albumin are released. These binding spots on albumin open for ionized calcium to bind, which reduces the amount of physiologically active calcium. Hypercalcemia and hypercalciuria increase the incidence of calcium stones, by increasing the urinary saturation of calcium salts and by binding negative-

Table 4. Clinical Parameter Groups and Corresponding Biochemical and Epidemiological Variables With Corresponding Correlation Values

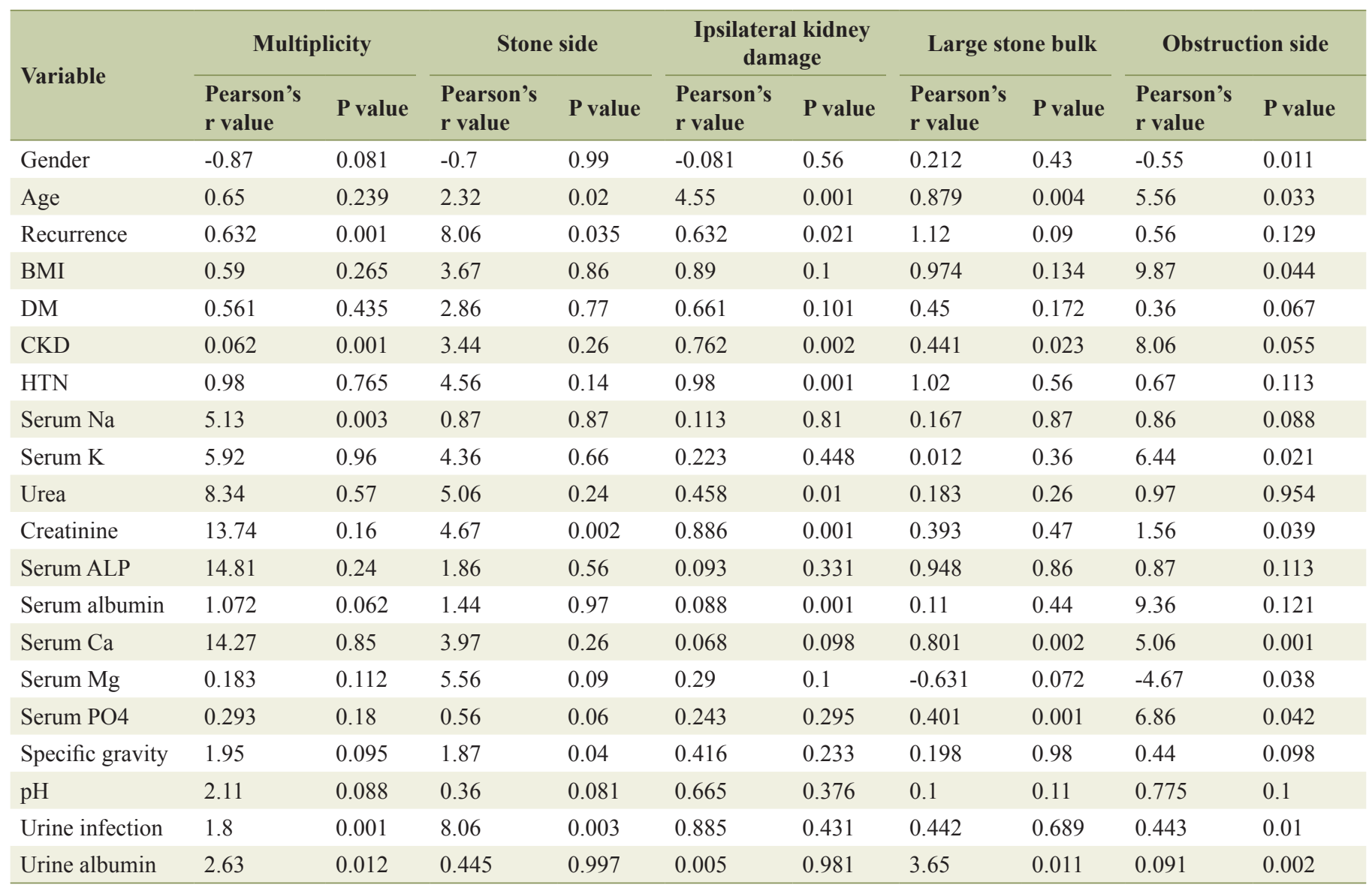


ly charged inhibitors of stone formation [8]. Increased level of serum calcium should lead to analysis of both ionized calcium and intact PTH with the aim to button in on patients who might have undiagnosed hyperparathyroidism [9]. A study [10] stated that stone patients have a relative risk of hypercalcemia and hypercalciuria nine to 18 times more than non-stone formers, respectively. Nonetheless, by definition of hypercalcemia (serum calcium $>2.75 \mathrm{mmol} / \mathrm{L}$ ), only two patients $(0.03 \%$ ) in our cohort were diagnosed as real hypercalcemia, which consistent with the research of a prospective study [11]. In a large prospective study, calcium intake was measured and high dietary intake was inversely correlated with kidney stone risk (relative risk: 0.56) [12]. In another large prospective cohort study of men, the relative risk of stone formation for highest and lowest quintiles of calcium intake was 0.69 [13]. Higher calcium intake decreased the risk of stones in females by up to $28 \%$ in a prospective cohort study [14]. Some studies indicated that serum phosphate level was a significant risk factor of calcium urolithiasis $[15,16]$. The renal phosphate leak theory explains calcium nephrolithiasis in recurrent stone cases by implicating that the defect of renal tubules will prevent phosphate reabsorption [17]. The result is hyperphosphaturia, with low serum phosphate level, causing an increase in the GIT absorption of calcium, which results in an increased renal load of calcium resulting in hypercalciuria. Nonetheless, the value of renal phosphate leak theory in the formation of calcium nephrolithiasis is questionable. Few studies showed no consistent compelling association of patient's serum phosphate level with stone recurrence [18].

When the urinary parameters of patients with idiopathic hypercalciuria were equated to those with normocalciuria, patients with former were found to have increased urinary sodium and sodium intake [19]. In a randomized study comparing low calcium and sodium diet and animal protein, the low sodium and animal protein diet resulted in fewer stone recurrences [20]. Increased sodium diet was related to $61 \%$ increase in nephrolithiasis risk in a large prospective study of women [21]. In a randomized study of 210 patients with hypercalciuria with calcium stones, a low sodium diet culminated in lower urinary sodium, as well as lower urinary calcium and oxalate excretion and resulted in normalization of urine calcium excretion for one third of patients [22].

Negative correlation between upper urinary tract obstruction side number and serum magnesium was observed in our series. Some authors indicated that magnesium can lower the risk of stone formation by diverse mechanisms. Magnesium chelates urinary oxalate, producing a soluble magnesium-oxalate complex than calcium oxalate and thus increases urinary citrate level [23]. A low urinary magnesium level has been seen in calcium stone formers with hypercalciuria. Clinically, oral supplementation of magnesium citrate increases urinary magnesium and citrate excretion. Magnesium supplement in deficient patients proved to decrease the recurrence rate of urolithiasis [24].

Serum potassium was observed to be positively correlated with upper urinary tract obstruction side number. Upper urinary tract obstruction invariably leads to hydronephrosis and renal insufficiency if ignored for long period of time. When renal function deteriorates, the ability to effectively regulate serum potassium via the $\mathrm{Na}^{+} / \mathrm{K}^{+}$-ATPase and multiple other mechanisms declines significantly. Although atoning mechanism exists, serum potassium will elevate continuously after it reaches its limits.

Our analysis showed that older patients were correlated with larger stone volume, higher risk of bilateral upper urinary tract calculi and obstruction, and severe ipsilateral kidney damage. Past studies have proved that older patients with stone formation had more condition related to metabolic syndromes than younger patients $[25,26]$. The clubbing between metabolic syndrome and nephrolithiasis has been established by some past studies $[27,28]$. One different scenario leading to the aforementioned condition is the steady decline in renal function that occurs with advanced age, as super-saturation and nephrolithiasis have been attributed to renal tubular cell damage [29]. Furthermore, older patients inclined to have more peculiar presentations of urolithiasis, which cause delay in the diagnosis and management [30]. This may explain why bilateral upper urinary tract calculi and larger stone volume were more frequent in older patients. In conclusion, older patients with urolithiasis usually have larger and more complex stone disease. A random spot urine sample was used for urine culture to establish organisms producing urease. The concomitant finding of high urine $\mathrm{pH}(>7.5)$ in few cases indicated that the patient might have formed an infection stone (magnesium ammonium phosphate + carbonate apatite). Low urine $\mathrm{pH}$ presents a risk for uric acid precipitation and subsequent stone formation [31].

In our study, urinary tract infection (UTI) was positively correlated with multiple stone, bilateral upper urinary tract stone and obstruction, and larger stone volume. UTI has been proved to be associated with the formation of stones, the most common composition being struvite (magnesium ammonium phosphate). Urease producing bacteria divides urea into ammonia, resulting in an alkaline urinary $\mathrm{pH}$ with subsequent struvite stone formation. Nevertheless, not all stones associated with UTI are composed of struvite and not all are associated with urea-splitting organisms [32, 33]. It is uncharted whether non-struvite infected stones result from infection itself or become secondary infected after formation. It has been observed that pathogenic bacteria from stones could initiate renal inflammation leading to crystal aggregation and subsequent nephrolithiasis. Moreover, the presence of bacteria could alter the local microenvironment by metabolic activity which further promotes lithogenesis $[34,35]$. Rather than calculating the total estimated GFR from serum creatinine, authors measured the spilt GFR through Tc-99m DTPA renal scan which enabled them to accurately evaluate the ipsilateral kidney damage level caused by nephrolithiasis. Thus, authors were able to identify the correlation between ipsilateral kidney damage, nephrolithiasis and various biochemical parameters.

In present study, older age, recurrence disease, hypertension (HTN) was positively correlated with the level of ipsilateral kidney damage. There is a proven firm association between HTN and stone formation, as numerous studies have pointed HTN as an independent predictor of nephrolithiasis [36]. Few studies suggest that dysregulation in renal calcium metabolism existed in patients with HTN, leading to increased hypercalciuria [37]. Additionally vice versa, in patients with 
nephrolithiasis, the incidence of HTN has been seen to be more than that of patients without nephrolithiasis [38]. It has been observed that uncontrolled hypertensive patients with nephrolithiasis have a greater risk for CKD as HTN is formidably associated with CKD. Recurrent stone formation history was proved to be significantly correlated with ipsilateral kidney damage. Amongst 171 patients with idiopathic calcium stones in a recent study [39], the 30 patients with reduced GFR (eGFR $<80 \mathrm{~mL} / \mathrm{min} / 1.73 \mathrm{~m}^{2}$ ) had significant incidence of recurrent disease with nearly one stone per patient per year.

In our study, negative correlation was observed between ipsilateral kidney damage and serum albumin level. Urine protein level was positively correlated with larger stone bulk and stone multiplicity. Although no correlation was observed between ipsilateral kidney damage and urine protein level, lower serum albumin level was frequently found in CKD patient mainly caused by proteinuria. It was observed in few studies that proteinuria was a surrogate outcome in CKD, with changes in proteinuria recommended as a surrogate for renal disease progression [40]. A study reported [1] atrophic kidney cortex $(<5 \mathrm{~mm})$, proteinuria $(>300 \mathrm{mg} / \mathrm{d})$, large stone bulk $\left(>1,500 \mathrm{~mm}^{2}\right)$, pediatric age group and recurrent UTI, were predictors of poor renal outcome in upper urinary tract stone formation patients with CKD. This goes hand in hand with our observations. The authors identify several limitations of the current study. We did not categorize patients according to stone compositions because they were not regularly indentified in our cohort. The invaluable $24 \mathrm{~h}$ urine analysis for super-saturation profile, which includes urinary calcium, phosphate, citrate, oxalate, etc., was also not involved in our study.

\section{Conclusions}

Our analysis demonstrated upper urinary tract obstruction was more likely to correlate with abnormal electrolyte metabolism in terms of serum calcium, magnesium and phosphate. Older age and UTI were positively associated with larger stone volume and complex stone patterns. Ipsilateral kidney damage associated with upper tract obstruction is correlated with old age, recurrence, hypertension and low serum albumin.

\section{References}

1. Moe OW. Kidney stones: pathophysiology and medical management. Lancet. 2006;367(9507):333-344.

2. Pak CY, Resnick MI, Preminger GM. Ethnic and geographic diversity of stone disease. Urology. 1997;50(4):504507.

3. Scales CD, Jr., Smith AC, Hanley JM, Saigal CS, Urologic Diseases in America P. Prevalence of kidney stones in the United States. Eur Urol. 2012;62(1):160-165.

4. Ansari MS, Gupta NP, Hemal AK, Dogra PN, Seth A, Aron M, Singh TP. Spectrum of stone composition: structural analysis of 1050 upper urinary tract calculi from northern India. Int J Urol. 2005;12(1):12-16.

5. Mandel EI, Taylor EN, Curhan GC. Dietary and lifestyle factors and medical conditions associated with urinary citrate excretion. Clin J Am Soc Nephrol. 2013;8(6):901908.

6. Mishra S, Sinha L, Ganesamoni R, Ganpule A, Sabnis RB, Desai M. Renal deterioration index: preoperative prognostic model for renal functional outcome after treatment of bilateral obstructive urolithiasis in patients with chronic kidney disease. J Endourol. 2013;27(11):14051410.

7. Antonelli JA, Maalouf NM, Pearle MS, Lotan Y. Use of the National Health and Nutrition Examination Survey to calculate the impact of obesity and diabetes on cost and prevalence of urolithiasis in 2030. Eur Urol. 2014;66(4):724-729.

8. Skolarikos A, Straub M, Knoll T, Sarica K, Seitz C, Petrik A, Turk C. Metabolic evaluation and recurrence prevention for urinary stone patients: EAU guidelines. Eur Urol. 2015;67(4):750-763.

9. Pearle MS, Goldfarb DS, Assimos DG, Curhan G, Denu-Ciocca CJ, Matlaga BR, Monga M, et al. Medical management of kidney stones: AUA guideline. J Urol. 2014;192(2):316-324.

10. Goldfarb DS, Arowojolu O. Metabolic evaluation of firsttime and recurrent stone formers. Urol Clin North Am. 2013;40(1):13-20.

11. Maalouf N. Approach to the adult kidney stone former. Clin Rev Bone Miner Metab. 2012;10(1):38-49.

12. Kadlec AO, Turk TM. Update on the evaluation of repeated stone formers. Curr Urol Rep. 2013;14(6):549-556.

13. Eisner BH, Sheth S, Dretler SP, Herrick B, Pais VM, Jr. Abnormalities of 24-hour urine composition in first-time and recurrent stone-formers. Urology. 2012;80(4):776779 .

14. Koyuncu HH, Yencilek F, Eryildirim B, Sarica K. Family history in stone disease: how important is it for the onset of the disease and the incidence of recurrence? Urol Res. 2010;38(2):105-109.

15. Pak CY, Peterson R, Poindexter JR. Adequacy of a single stone risk analysis in the medical evaluation of urolithiasis. J Urol. 2001;165(2):378-381.

16. Parks JH, Goldfisher E, Asplin JR, Coe FL. A single 24hour urine collection is inadequate for the medical evaluation of nephrolithiasis. J Urol. 2002;167(4):1607-1612.

17. Healy KA, Hubosky SG, Bagley DH. 24-hour urine collection in the metabolic evaluation of stone formers: is one study adequate? J Endourol. 2013;27(3):374-378.

18. Nayan M, Elkoushy MA, Andonian S. Variations between two 24-hour urine collections in patients presenting to a tertiary stone clinic. Can Urol Assoc J. 2012;6(1):30-33.

19. Lee TT, Elkoushy MA, Andonian S. Are stone analysis results different with repeated sampling? Can Urol Assoc J. 2014;8(5-6):E317-322.

20. Hosking DH, Erickson SB, Van den Berg CJ, Wilson DM, Smith LH. The stone clinic effect in patients with idiopathic calcium urolithiasis. J Urol. 1983;130(6):11151118.

21. Jeong IG, Kang T, Bang JK, Park J, Kim W, Hwang SS, Kim HK, et al. Association between metabolic syndrome and the presence of kidney stones in a screened popula- 
tion. Am J Kidney Dis. 2011;58(3):383-388.

22. Khan SR, Canales BK. Genetic basis of renal cellular dysfunction and the formation of kidney stones. Urol Res. 2009;37(4):169-180.

23. Dursun M, Ozbek E, Otunctemur A, Sahin S, Cakir SS. Clinical presentation of urolithiasis in older and younger population. Arch Ital Urol Androl. 2014;86(4):249-252.

24. de Cogain MR, Lieske JC, Vrtiska TJ, Tosh PK, Krambeck AE. Secondarily infected nonstruvite urolithiasis: a prospective evaluation. Urology. 2014;84(6):1295-1300.

25. Tavichakorntrakool R, Prasongwattana V, Sungkeeree S, Saisud P, Sribenjalux P, Pimratana C, Bovornpadungkitti $\mathrm{S}$, et al. Extensive characterizations of bacteria isolated from catheterized urine and stone matrices in patients with nephrolithiasis. Nephrol Dial Transplant. 2012;27(11):4125-4130.

26. Losito A, Nunzi EG, Covarelli C, Nunzi E, Ferrara $\mathrm{G}$. Increased acid excretion in kidney stone formers with essential hypertension. Nephrol Dial Transplant. 2009;24(1):137-141.

27. Eisner BH, Porten SP, Bechis SK, Stoller ML. Hypertension is associated with increased urinary calcium excretion in patients with nephrolithiasis. J Urol. 2010;183(2):576579.

28. Gillen DL, Coe FL, Worcester EM. Nephrolithiasis and increased blood pressure among females with high body mass index. Am J Kidney Dis. 2005;46(2):263-269.

29. Herrel L, Pattaras J, Solomon T, Ogan K. Urinary stone risk and cola consumption. Urology. 2012;80(5):990-994.

30. Shuster J, Jenkins A, Logan C, Barnett T, Riehle R, Zackson $\mathrm{D}$, Wolfe $\mathrm{H}$, et al. Soft drink consumption and urinary stone recurrence: a randomized prevention trial. J Clin Epidemiol. 1992;45(8):911-916.

31. Wang X, Xu X, Wu J, Zhu Y, Lin Y, Zheng X, Xie L. Sys- tematic review and meta-analysis of the effect of alcohol intake on the risk of urolithiasis including dose-response relationship. Urol Int. 2015;94(2):194-204.

32. Ferraro PM, Taylor EN, Gambaro G, Curhan GC. Caffeine intake and the risk of kidney stones. Am J Clin Nutr. 2014;100(6):1596-1603.

33. Ferraro PM, Taylor EN, Gambaro G, Curhan GC. Soda and other beverages and the risk of kidney stones. Clin J Am Soc Nephrol. 2013;8(8):1389-1395.

34. Wang S, Zhang Y, Mao Z, He X, Zhang Q, Zhang D. A meta-analysis of coffee intake and risk of urolithiasis. Urol Int. 2014;93(2):220-228.

35. Penniston KL, Steele TH, Nakada SY. Lemonade therapy increases urinary citrate and urine volumes in patients with recurrent calcium oxalate stone formation. Urology. 2007;70(5):856-860.

36. McCauley LR, Dyer AJ, Stern K, Hicks T, Nguyen MM. Factors influencing fluid intake behavior among kidney stone formers. J Urol. 2012;187(4):1282-1286.

37. Taylor EN, Stampfer MJ, Curhan GC. Dietary factors and the risk of incident kidney stones in men: new insights after 14 years of follow-up. J Am Soc Nephrol. 2004;15(12):3225-3232.

38. Penniston KL, Nakada SY. Effect of dietary changes on urinary oxalate excretion and calcium oxalate supersaturation in patients with hyperoxaluric stone formation. Urology. 2009;73(3):484-489.

39. Straub DA. Calcium supplementation in clinical practice: a review of forms, doses, and indications. Nutr Clin Pract. 2007;22(3):286-296.

40. Borghi L, Meschi T, Amato F, Briganti A, Novarini A, Giannini A. Urinary volume, water and recurrences in idiopathic calcium nephrolithiasis: a 5-year randomized prospective study. J Urol. 1996;155(3):839-843. 